\title{
Impact of anterior skull base fracture on lateralized olfactory function*
}

\author{
V. Gudziol'1,2, T. Marschke ${ }^{3}$, J. Reden', T. Hummel' \\ Department of Otorhinolaryngology, Smell and Taste Clinic, "Technische Universität Dresden, Germany \\ 2Department of Otorhinolaryngology, Head and Neck Surgery, Municipal Hospital Dresden, Germany \\ 3 Department of Anesthesiology, "Technische Universität Dresden", Germany
}

Rhinology 58: 1, 45 - 50, 2020

https://doi.org/10.4193/Rhin18.092

*Received for publication:

May 4, 2018

Accepted: May 11, 2019

\begin{abstract}
Background: Data on the impact of anterior skull base fractures (aSBF) on lateralized olfactory function are missing. The goal of the study was to investigate olfactory function in patients with traumatic brain injury (TBI) due to aSBF separately for each side and assess the frequency of lateralized smell impairment.
\end{abstract}

Methods: Retrospective, single center study of olfactory function in 93 patients with aSBF. Olfactory function was assessed by means of the Sniffin' Sticks test battery for each side of the nose, separately. TBI severity was graded according to the Glasgow Coma Scale. Average time interval between olfactory test and trauma was 6.5 years. General olfactory function was defined as the best side out of both nostrils.

Results: A total of 50 patients had unilateral and 43 patients bilateral aSBF. The grade of TBI was inversely correlated with olfactory function. General olfactory function was significantly worse in patients with bilateral aSBF compared to patients with unilateral aSBF. Clinically significant side by side differences in olfactory function were found in 18 and $30 \%$ respectively for unilateral and bilateral aSBF. Grade of TBI had no significant impact on side differences. Among patients with unilateral aSBF olfactory function was not significantly different between the fractured and the non-fractured side.

Conclusion: The severity of TBI and bilateral more than unilateral aSBF results in more impaired olfactory function. Lateralized olfactory deficits were not more frequent in any group, regardless of the fracture type and side.

Key words: anterior skull base fracture, olfaction, skull base , trauma, traumatic brain injury

\section{Introduction}

Although there exist abundant data on smell loss due to traumatic brain injury (TBI), data on olfactory function among patients with TBI and anterior skull base fractures (aSBF) are rare (for review compare Schofield) ${ }^{(1)}$. Some authors maintain that smell loss following TBI is a result of damage to the olfactory pathway close to the skull base e.g. scarring in the olfactory cleft, shearing of the olfactory nerves at the cribriform plate, anterior skull base fractures or contusion of the olfactory bulb ${ }^{(2-4)}$. Other authors suggest that TBI results in damage of olfactory eloquent brain structures centrally to the olfactory bulb ${ }^{(5)}$. aSBF is the result of immense forces that lead to disruption of the bony skull base close to the olfactory pathway.

Ipsilateral smell loss following aSBF would support the hypothesis that aSBF has an additional impact on smell loss among patients with TBI. To our knowledge data on the lateralized impact of anterior skull base fractures on olfactory function are missing. Two study groups investigated olfactory function in patients with TBI some of whom had additional aSBF. Joung et al. did not find a significant impact of aSBF on olfactory function ${ }^{(6)}$. Haxel and coauthors identified two patients with aSBF among 7 posttraumatic anosmic patients ${ }^{(7)}$. Neither Joung et al. nor Haxel et al. investigated olfactory function separately for each side. Evaluation of olfactory function especially following head 
injury is complicated by the course of the olfactory signal pathway that runs ipsilaterally from the olfactory epithelium to the olfactory bulb, along the olfactory tract up to the olfactory cortex ${ }^{(8,9)}$. Head trauma resulting in a unilateral disruption of the olfactory signal pathway may lead to ipsilateral smell loss while the contralateral side might demonstrate a normal sense of smell. In case of normal olfactory function of the contralateral side, birhinal olfactory testing is unlikely to unveil unilateral olfactory loss ${ }^{(2-4)}$.

Information about lateralized differences of olfactory function in patients following traumatic brain injury is rare. Clinically significant side differences are present in $39 \%$ of patients with self-reported olfactory dysfunction following head trauma ${ }^{(10)}$. Unilateral mechanical intranasal obstructions, shearing of olfactory nerves at the cribriform plate or damage to the olfactory bulbs have been discussed as possible causes for asymmetric olfactory deterioration following TBI ${ }^{(10-12)}$. On the other hand, damage to cerebral olfactory eloquent structures may lead to birhinal olfactory loss ${ }^{(11,13-15)}$. Additionally, diffuse axonal injury due to TBI can lead to white matter atrophy with consecutive smell loss ${ }^{(16)}$.

Aim of the present study was to investigate whether TBI with anterior skull base fractures close to the olfactory signal pathway results in ipsi- or bilateral smell loss. Ipsilateral smell loss in case of unilateral anterior skull base fracture would support the hypothesis of a lesion peripheral from the olfactory tract. The antithesis would be that unilateral anterior skull base fracture results in bilateral smell loss. That finding would support the idea of a more central damage of the olfactory signal pathway. To that end olfactory function was measured separately for each nostril among patients who sustained TBI with unilateral and bilateral anterior skull base fractures.

The selected patients had anterior skull base fractures involving the osseous walls of the nasal cavity and the paranasal sinus. All patients received surgical skull base exploration with closure of the fracture lines in order to prevent dura-laceration-related intracranial complications. Indication for skull base reconstruction differs widely among reference rhinology centers. Several experts recommend skull base reconstruction in case of rhinoliquorrhea ${ }^{(17,18)}$. Others favor conservative therapy in cases with self-limiting rhinoliquorrhea ${ }^{(18,19)}$. A London-based group recommended surgery in patients with intracranial air or displacement of the fracture by more than the thickness of the bone ${ }^{(20)}$. Yet another center advocates skull base exploration without clear clinical and radiological signs of dura laceration ${ }^{(21)}$. Schoentgen and co-authors conclude that, at present, there is no common consensus for diagnosis and treatment of anterior skull base fractures ${ }^{(19)}$.

\section{Patients and methods}

This single-center retrospective study was approved by the
Figure 1. Flow chart of the investigated patients; number of patients with unilateral and bilateral fractures of the skull base.

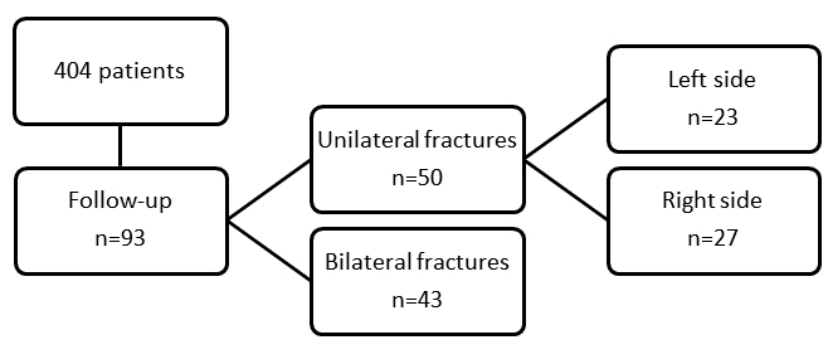

Ethics Board at the Medical Faculty of the TU Dresden. Patients provided written informed consent to participate in the study. Patients with TBI and fractures of the skull base were investigated using computed tomography of the skull. Electronic reports of the Department of Otorhinolaryngology at the TU Dresden were screened for the German key words indicating anterior skull base fracture for a 10 years period (October 1998 to October 2008). Patients were included when they received surgery for aSBF (skull base fracture involving the nasal cavity and/ or paranasal sinuses), when they were age 18 or older at time of follow up and when they were cooperative to answer the questionnaires and perform olfactory tests. Those patients who had other causes for damage of the anterior skull base e.g. as a result of endoscopic sinus surgery or patients with spontaneous skull base fistulas were excluded from the study.

All eligible patients were contacted either by mail or by phone to participate in a follow-up examination. Follow-up examination was performed from November 2011 to August 2012 in the out-patients clinic of the Department of Otorhinolaryngology, Dresden Medical School. Medical files of all participants were reviewed for the localization of the anterior skull base fracture. Physical examination included nasal endoscopy to assess chronic rhinosinusitis, mucoceles and endonasal scarring. A validated olfactory test was applied separately for each nostril for assessment of olfactory function.

\section{Patients}

A total of 414 patients with anterior skull base fractures could be identified in our database; 404 patients fulfilled all inclusion and none of the exclusion criteria; 93 patients were available for follow-up examination (Figure 1).

\section{Olfactory test}

Olfactory function was evaluated separately for each nostril. The side which was tested first was randomly selected. For assessment of olfactory function pen-like odor dispensing devices ('Sniffin' Sticks') were employed (22,23). This kit is comprised of three subtests of olfactory function, namely tests for phenyl 
Table 1. Number of patients who demonstrated CT-morphological fracture lines in specific areas of the skull base; notice: multiple sinus can be involved per patient.

\begin{tabular}{|lccc|}
\hline & Frontal sinus & Ethmoid sinus & Sphenoid sinus \\
\hline Unilateral right & 13 & 14 & 11 \\
\hline Unilateral left & 12 & 10 & 8 \\
\hline Bilateral right & 21 & 34 & 14 \\
\hline Bilateral left & 19 & 32 & 16 \\
\hline
\end{tabular}

Table 2. Self-evaluation of olfactory function.

Number of patients

\begin{tabular}{|lc|}
\hline Complete loss & Number of patients \\
\hline Deteriorated & $16(17.2 \%)$ \\
\hline Normal & $21(22.6 \%)$ \\
\hline Better than normal & $50(53.8 \%)$ \\
\hline Excellent & $5(5.4 \%)$ \\
\hline
\end{tabular}

ethyl alcohol odor threshold (T), odor discrimination (D), and odor identification (I). For a more comprehensive analysis results from the three tests were summed up to a composite score, the so-called 'TDI-score'.

General olfactory function is represented by the olfactory function of the best nostril (24). The olfactory test result of the best nostril was used to describe overall olfactory function. The patients' smell function was assigned to the categories of normosmia, hyposmia or functional anosmia ${ }^{(22)}$.

\section{Traumatic brain injury}

Grade of TBI was obtained from the patients' files. Grading of TBI was based on the Glasgow Coma Scale (GCS) (I': minor, GCS:1315; $I^{\circ}$ : moderate, GCS:9-12 and $\mathrm{III}^{\circ}$ : severe, GCS:3-8) ${ }^{(25)}$.

\section{Statistics}

Data were investigated using SPSS 25.0 for Windows ${ }^{\mathrm{TM}}$ (SPSS Inc., Chicago, IL, USA). T-tests for paired samples were used to compare lateralized results within a group. T-tests for independent samples were used to compare results between the studied groups. An alpha-level of 0.05 was regarded to be significant.

\section{Results}

As mentioned above, 404 patients could be identified who fulfilled all criteria for participation (Figure 1). Ninety-three were available for follow-up examination; 50 had a unilateral fracture of the anterior skull base, 43 had a bilateral fracture. Time between TBI and follow-up examination was on average 6.4 years (range 2.2 to 13.9 years). Evaluation of olfactory function could be performed in 23 patients with a unilateral fracture on the left side and in 27 patients with a unilateral fracture on the right side. In 43 patients olfaction was measured in patients with a bilateral anterior skull base fracture. Thirty-nine men and 11 women were in the unilateral fracture group (mean age 44 \pm 15.8 years) and 38 men and 5 women (mean age $42 \pm 13.3$ years) in the bilateral fracture group. The extent of the fractured skull base is indicated in Table 1.

Self-evaluation of olfactory function revealed a normal or better than normal sense of smell in 56 patients. Thirty-seven patients indicated smell loss (Table 2). Measurement of general olfac- tory function (TDI-score of the best side) showed 18, 53 and 22 patients to be normosmic, hyposmic and functionally anosmic, respectively.

In 75 cases the TBI grade could be obtained from the patients' files (Table 3). TBI grades were significantly higher in the bilateral group $\left(\chi^{2}=28.8, d f=4, p=0.001\right)$.

General olfactory function (TDI-score of the best side) was the lower the higher the grade of the TBI $(F[2,74]=2.5, p<0.05$, Figure 2 ). In addition, general olfactory function was significantly lower $(p=0.01)$ in the group with bilateral fractures (mean \pm standard deviation; $20.4 \pm 8.1$ ) compared to the group with unilateral fractures $(24.5 \pm 7.0)$.

In the group with unilateral fractures no significant difference $(p=0.10)$ was observed between the fractured side $(22.3 \pm 7.4)$ compared to the non-fractured side $(23.4 \pm 7.0)$. Localization of the fracture (frontal sinus, ethmoid, sphenoid sinus) had no significant impact on olfactory function (TDI-score of the fractured side) neither on the right or the left side (Table 4).

When looking for side differences among the unilateral fracture group, 7 patients demonstrated clinically significant lower olfactory function (by 5.5 and more points of the TDI-score) on the fractured side ${ }^{(26)}$. In contrast, 2 patients had a clinically significant increased olfactory function on the fractured side. In the remaining 41 patients (82\%) no such differences were observed. In the bilateral fractured group, a clinically significant side difference was found in 13 patients (30\%). There was no significant effect of the TBI grade on olfactory side differences $(F[2,74]=1.03 ; p=0.36)$.

Nasal endoscopy revealed scarring or synechia that might block the airflow to the olfactory epithelium in 8 patients with unilateral fractures and in 4 and 2 patients with bilateral fractures on the right and the left side, respectively. No patient demonstrated bilateral scarring or synechia in the nasal cavity. Olfactory function was significantly better $(p<0.01)$ on the open side (TDI-score $21.8 \pm 7.6$ ) compared to the scarred side (TDI-score $11.3 \pm 9.7)$.

\section{Discussion}

In this study olfactory function was assessed using a validated 
Figure 2. General Olfactory function of the better nostril in relation to the grade of TBI.

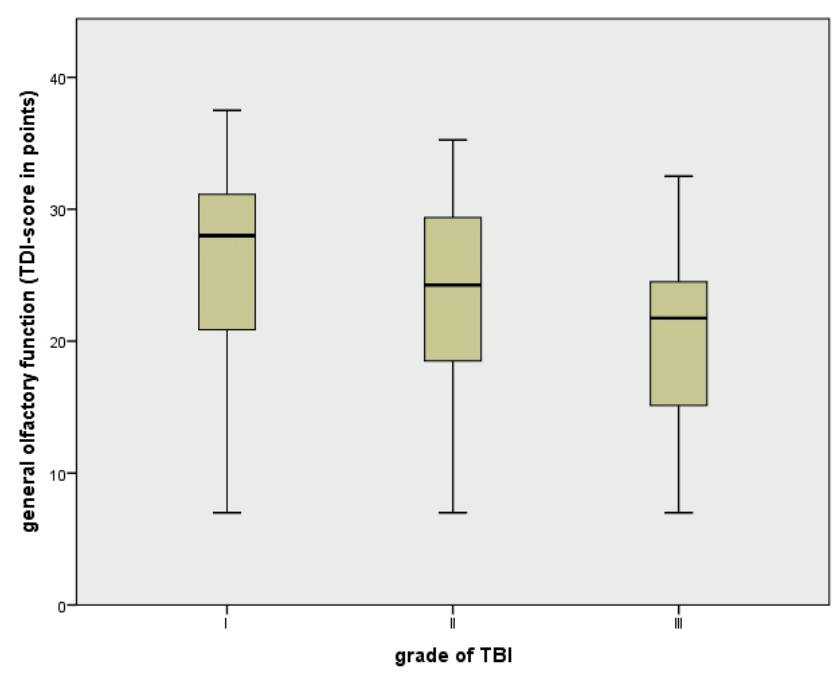

Table 3. Number of patients in relation to the grade of TBI and the fracture type.

\begin{tabular}{|cccc|} 
& \multicolumn{1}{c}{ TBI $^{\circ}$} & TBI II $^{\circ}$ & TBI III $^{\circ}$ \\
\hline unilateral & $15(37.5 \%)$ & $11(27.5 \%)$ & $14(35.0 \%)$ \\
bilateral & $9(25.7 \%)$ & $8(22.9 \%)$ & $18(51.4 \%)$ \\
\hline
\end{tabular}

Table 4. p-values of univariate analysis of variance of olfactory function between the involved fractured sinuses separately for each side.

\begin{tabular}{|lccc} 
& Frontal sinus & Ethmoid sinus & Sphenoid sinus \\
\hline Frontal sinus & & $\mathrm{p}=0.73$ right & $\mathrm{p}=0.44$ right \\
Ethmoid sinus & $\mathrm{p}=0.99$ left & & $\mathrm{p}=0.70$ right \\
Sphenoid sinus & $\mathrm{p}=0.67$ left & $\mathrm{p}=0.46$ left & \\
\hline
\end{tabular}

test separately for each side. At the time of follow-up examination none of the participants indicated any claim for compensation or medical disability certification. Importantly, the prevalence of smell loss in that study was evaluated among participants who did not ask for smell assessment because of self-reported olfactory loss.

The rate of self-reported smell loss in patients with a history of TBI varies between 30 and $100 \%{ }^{(27,28)}$. In the present investigation, $40 \%$ of the patients indicated a decreased olfactory function. In contrast to the studies by Doty et al. and Green et al., the present population volunteered in the study and was not investigated because of self-reported olfactory loss or for any assessment that could result in any form of compensation or certification of medical disability ${ }^{(27,28)}$.

In the present study, $60 \%$ of the patients evaluated their sense of smell as normal or as better than normal. This is in contrast to the olfactory test results that indicate normosmia in only $19 \%$. A comparable discrepancy between self-evaluated and measured olfactory function was reported in patients with TBI previously (29). This discrepancy again illustrates the poor reliability of self-evaluation of olfactory function and underlines the need of olfactory testing using validated tests $(2,30)$.

The current study provides evidence that olfactory function decreases with an increasing grade of TBI which confirms previous studies ${ }^{(29,31)}$. Distortion of olfactory function is more likely following severe head trauma. Among the investigated patients the precise localization of the disturbance within the olfactory signal pathway cannot be determined. However, the present study confirms the idea that head trauma itself produces smell loss due to effects downstream the olfactory pathway. If the main effect would be at the level of the cribriform plate more lateralized differences in olfactory function could have been expected - which was not the case. This supports the idea that TBI-related olfactory loss is the result of damage of olfactory eloquent brain structures. What is adding to this idea is work on the prediction of the severity of TBI-related olfactory loss which is possibly based on the pattern of brain lesions ${ }^{(32)}$. Olfactory function in patients with bilateral fractures was significantly poorer than in patients with unilateral fractures. That figure can be explained by the higher grades of TBI in the group with bilateral anterior skull base fractures. Remarkably, unilateral fracture of the skull base did not result in a significant ipsilateral smell loss compared to the contralateral side. That observation also supports the hypothesis that TBI itself rather than peripheral disruption of the olfactory signal pathway is the cause of smell loss in the majority of patients in this group. Additionally, it shows that the performed surgical management of the anterior skull base facture had no major impact on olfactory function. In fact, based on nasal endoscopy significantly reduced olfactory function was found on the scarred side of the nose.

Although there was no statistically significant side difference of olfactory function among the group of patients with unilateral anterior skull base fracture, $18 \%$ demonstrate clinically significant side differences ${ }^{(26)}$. This was in line with previous work showing that $15 \%$ of healthy subjects exhibit side differences (33). Interestingly, $30 \%$ of the patients with a bilateral fractured skull base had clinically significant side differences. That number corresponds to a previous report with side differences in 39\% patients with post-traumatic smell loss ${ }^{(10)}$. One possible explanation for the higher rate of side differences among the bilateral compared to the unilateral fractured group might be the (overall) lower olfactory function among the "bilateral" patients. In fact, more side differences are found among patients with smell 
loss compared to normosmic individuals ${ }^{(33)}$. Another explanation could be that TBI itself affects the olfactory signal pathway asymmetrically due to the unilaterality of the olfactory signal pathway. No significant impact of the grade of TBI on olfactory side differences could be found in the present study. Previous research emphasized that unilateral smell loss is a predictor for global olfactory loss within the next 5 years which prompts the question whether especially the studied patients with unilateral smell loss are at risk to develop global olfactory loss in the near future ${ }^{(34)}$. The current data on the missing correlation between functional side differences in relation to the side of the impact also seem to indicate that the olfactory deficit is not due to a rupture of olfactory fibers at the level of the cribriform plate, but that it is more the overall impact of the trauma on the brain which determines the impact of the blow on olfactory function (11).

Investigating the diverse locations of the fractured skull base did not reveal significant differences. It has to be noted that a possible individual fracture of the cribriform plate, that likely results in a disruption of the olfactory nerves, was not separately recorded in the present study. All patient's skull bases were surgically explored and closed in order to prevent ascending meningitis. One could assume that surgery exclusively in the frontal sinus with an external approach is less likely to cause disruption of the olfactory epithelium in the nasal cavity than transnasal surgery in the ethmoid or the sphenoid sinus. Fortunately, our surgical approach seems not to impact on olfactory function. That mes- sage is also underlined by the good and symmetrical olfactory function among the patients with unilateral fractured anterior skull base.

\section{Conclusion}

Higher grades of TBI are associated with bilateral olfactory loss. Unilateral fractures of the anterior skull base and the localization of the fractures do not impact on ipsilateral olfaction supporting the hypothesis that bilateral damage of olfactory-eloquent brain structures is causative for posttraumatic smell loss, at least in many cases. Notably to say that on an individual level e.g. scarring in the nasal cavity might result in lateralized differences in olfactory function. Clinically significant side differences of olfaction are found especially among patients with bilateral fractures what can be explained by generally poorer olfactory function among these patients.

\section{Author contribution}

VG, TM and TH designed the study, VG and TM did acquisition of data, all authors were involved in analysis and interpretation of data, in drafting the manuscript or revising it critically for important intellectual content and gave final approval of the version to be published.

\section{Conflict of interest}

The authors state no conflict of interests.

\section{References}

1. Schofield PW, Moore TM, Gardner A. Traumatic brain injury and olfaction: a systematic review. Front Neurol. 2014;5.

2. Landis BN, Hummel T, Hugentobler M, Giger R, Lacroix JS. Ratings of overall olfactory function. Chem Senses. 2003 Oct;28(8):6914.

3. Klimek L, Hummel T, Moll B, Kobal G, Mann WJ. Lateralized and bilateral olfactory function in patients with chronic sinusitis compared with healthy control subjects. Laryngoscope. 1998 Jan;108(1 Pt 1):111-4.

4. Betchen SA, Doty RL. Bilateral detection thresholds in dextrals and sinistrals reflect the more sensitive side of the nose, which is not lateralized. Chem Senses. 1998;23(4):453-7.

5. Fujiwara E, Schwartz ML, Gao F, Black SE, Levine B. Ventral frontal cortex functions and quantified MRI in traumatic brain injury. Neuropsychologia. 2008 Jan 31;46(2):46174.

6. Joung YI, Yi HJ, Lee SK, Im TH, Cho SH, Ko Y. Posttraumatic anosmia and ageusia: Incidence and recovery with relevance to the hemorrhage and fracture on the frontal base. J Korean Neurosurg S. 2007 Jul:42(1):1-5.

7. Haxel BR, Grant L, Mackay-Sim A. Olfactory dysfunction after head injury. J Head Trauma Rehabil. 2008 Nov-Dec;23(6):407-13.

8. Powell TP, Cowan WM, Raisman G. The central olfactory connexions. J Anat. 1965 Oct;99(Pt 4):791-813.

9. Savic I. Processing of odorous signals in humans. Brain Res Bull. 2001 Feb;54(3):30712.

10. Welge-Lussen A, Hilgenfeld A, Meusel T, Hummel T. Long-term follow-up of posttraumatic olfactory disorders. Rhinology. 2012 Mar;50(1):67-72.

11. Lotsch J, Reither N, Bogdanov V, Hahner A, Ultsch A, Hill K, et al. A brain-lesion pattern based algorithm for the diagnosis of posttraumatic olfactory loss. Rhinology. 2015 Dec;53(4):365-70.

12. Reiter ER, DiNardo LJ, Costanzo RM. Effects of head injury on olfaction and taste. Otolaryngol Clin North Am. 2004 Dec;37(6):1167-84.

13. Yousem DM, Geckle RJ, Bilker WB, Kroger $H$, Doty RL. Posttraumatic smell loss: relationship of psychophysical tests and volumes of the olfactory bulbs and tracts and the temporal lobes. Acad Radiol. 1999;6(5):264-72.

14. Yousem DM, Geckle RJ, Bilker WB, McKeown DA, Doty RL. Posttraumatic olfactory dysfunction: MR and clinical evaluation. AJNR Am J Neuroradiol. 1996 Jun-Jul;17(6):1171-9.
15. Gentry LR, Godersky JC, Thompson B. MR imaging of head trauma: review of the distribution and radiopathologic features of traumatic lesions. AJR American Journal of Roentgenology. 1988 Mar;150(3):663-72.

16. Gentry LR, Thompson B, Godersky JC. Trauma to the corpus callosum: MR features. AJNR American Journal of Neuroradiology. 1988 Nov-Dec;9(6):1129-38.

17. Bernal-Sprekelsen M, Alobid I, Mullol J, Trobat F, Tomas-Barberan M. Closure of cerebrospinal fluid leaks prevents ascending bacterial meningitis. Rhinology. 2005 Dec;43(4):277-81.

18. Piccirilli M, Anichini G, Cassoni A, Ramieri V, Valentini V, Santoro A. Anterior cranial fossa traumas: clinical value, surgical indications, and results-a retrospective study on a series of 223 patients. J Neurol Surg B Skull Base. 2012 Aug;73(4):265-72.

19. Schoentgen C, Henaux PL, Godey B, Jegoux F. Management of post-traumatic cerebrospinal fluid (CSF) leak of anterior skull base: 10 years experience. Acta Otolaryngol. 2013 Sep;133(9):944-50.

20. Swinson BD, Jerjes W, Thompson G. Current practice in the management of frontal sinus fractures. J Laryngol Otol. 2004 Dec;118(12):927-32.

21. Elies W. [The present state of frontal skull- 
base surgery (author's transl)]. Laryngo Rhinol Otol (Stuttg). 1982 Jan;61 (1):42-7.

22. Hummel T, Kobal G, Gudziol H, Mackay-Sim A. Normative data for the "Sniffin' Sticks" including tests of odor identification, odor discrimination, and olfactory thresholds: an upgrade based on a group of more than 3,000 subjects. European Archives of Otorhinolaryngology. 2007 Mar;264(3):23743.

23. Hummel T, Sekinger B, Wolf SR, Pauli E, Kobal G. "Sniffin' sticks": olfactory performance assessed by the combined testing of odor identification, odor discrimination and olfactory threshold. Chem Senses. 1997 Feb;22(1):39-52.

24. Klimek L, Hummel T, Moll B, Kobal G, Mann WJ. Lateralized and bilateral olfactory function in patients with chronic sinusitis compared with healthy control subjects. Laryngoscope. 1998;108(1 Pt 1):111-4.

25. Rimel RW, Jane JA, Edlich RF. An injury severity scale for comprehensive management of central nervous system trauma. JACEP. 1979 Feb;8(2):64-7.

26. Gudziol V, Lotsch J, Hahner A, Zahnert T,
Hummel T. Clinical significance of results from olfactory testing. Laryngoscope. 2006 Oct;116(10):1858-63.

27. Green P, Rohling ML, Iverson GL, Gervais RO Relationships between olfactory discrimination and head injury severity. Brain Inj. 2003 Jun;17(6):479-96.

28. Doty RL, Yousem DM, Pham LT, Kreshak AA Geckle R, Lee WW. Olfactory dysfunction in patients with head trauma. Arch Neurol. 1997;54(9):1131-40

29. Gudziol V, Hoenck I, Landis B, Podlesek D, Bayn M, Hummel T. The impact and prospect of traumatic brain injury on olfactory function: a cross-sectional and prospective study. Eur Arch Otorhinolaryngol. 2014 Jun;271(6):1533-40

30. Hummel $T$, Whitcroft $K L$, Andrews $P$, Altundag A, Cinghi C, Costanzo RM, et al. Position paper on olfactory dysfunction. Rhinol Suppl. 2017 Mar;54(26):1-30.

31. Callahan CD, Hinkebein J. Neuropsychological significance of anosmia following traumatic brain injury. J Head Trauma Rehabil. 1999 Dec;14(6):581-7.

32. Lotsch J, Ultsch A, Eckhardt M, Huart C,
Rombaux P, Hummel T. Brain lesion-pattern analysis in patients with olfactory dysfunctions following head trauma. Neuroimage Clin. 2016;11:99-105.

33. Gudziol V, Hummel C, Negoias S, Ishimaru $T$, Hummel $T$. Lateralized differences in olfactory function. Laryngoscope. 2007 May;117(5):808-11.

34. Gudziol V, Paech I, Hummel T. Unilatera reduced sense of smell is an early indicator for global olfactory loss. J Neurol. 2010 Jun:257(6):959-63.

Volker Gudziol, MD

ORL, Municipal Hospital

Friedrichstrasse 41

01067 Dresden

Germany

E-mail:

volker.gudziol@klinikum-dresden.de 